\title{
Multilinguales
}

\section{Analyse multimodale de l'ethos en interaction : le cas de l'interview politique télévisée}

Multimodal analysis of ethos in interaction: the case of televised political interview

تحليل متعدد الوسائط عن روح التفاعل:حالة المقابلة السياسية المتلفزة

\section{Zakaria Tanssaout}

\section{CpenEdition}

Journals

Édition électronique

URL : http://journals.openedition.org/multilinguales/1114

DOI : 10.4000/multilinguales. 1114

ISSN : 2335-1853

Éditeur

Université Abderrahmane Mira - Bejaia

Référence électronique

Zakaria Tanssaout, « Analyse multimodale de l'ethos en interaction : le cas de l'interview politique télévisée », Multilinguales [En ligne], 9 | 2018, mis en ligne le 01 juin 2018, consulté le 17 septembre 2019. URL : http://journals.openedition.org/multilinguales/1114; DOI : 10.4000/multilinguales.1114

Ce document a été généré automatiquement le 17 septembre 2019.

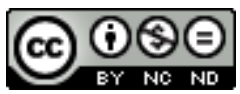

Multilinguales est mise à disposition selon les termes de la Licence Creative Commons Attribution Pas d'Utilisation Commerciale - Pas de Modification 4.0 International 


\title{
Analyse multimodale de l'ethos en interaction : le cas de l'interview politique télévisée
}

\author{
Multimodal analysis of ethos in interaction: the case of televised political \\ interview \\ تحليل متعدد الوسائط عن روح التفاعل:حالة المقابلة السياسية المتلفزة
}

\section{Zakaria Tanssaout}

1 L'objectif de l'interview politique télévisée consiste à faire découvrir aux téléspectateurs une personnalité politique en s'intéressant au projet qu'elle défend, à son idéologie, à sa vision de l'organisation de la société, etc. L'enjeu principal de l'interaction est donc de donner à voir une certaine image de l'homme politique interviewé. La construction de cette image s'effectue, comme dans toutes interactions verbales, à travers une négociation incessante entre les participants à l'interview. Afin de gagner l'assentiment des allocutaires (téléspectateurs), l'interviewé s'efforce de mettre en scène un ethos favorable. De son côté, l'intervieweur, par ses questions, contribue à façonner l'image de l'interviewé. Ceci dit, bien que l'interview fasse partie des genres de discours qui tendent vers la coopérativité, la mise en scène de l'ethos de l'interviewé ne se déroule pas toujours sans heurts. En effet, l'intervieweur vise parfois à «faire sortir le renard de sa tanière, en questionnant sur ce qui fait problème» (Nowakowska, 2012: 616). Ses questions visent, non seulement à faire parler l'homme politique, mais à discréditer son image publique et à saper sa crédibilité auprès des téléspectateurs. L'objectif de notre étude est précisément de rendre compte de cette coconstruction de l'ethos et de mettre au jour les modalités de sa mise en scène en situation d'interview politique télévisée. Il s'agit de décrire les images discursives attribuées par l'intervieweur à l'interviewé et celles que ce dernier revendique. Pour ce faire, l'analyse s'appuie sur un appareil conceptuel et méthodologique s'inspirant de l'Analyse du Discours en Interaction. Elle porte sur une interview de Nicolas Sarkozy (désormais NS), alors ministre de l'intérieur, réalisée par Jean Baptiste Prédali (JBP), journaliste et un des responsables du service «politique » de France 2. L'interaction 
dure huit minutes, et est extraite de l'émission 100 minutes pour convaincre diffusée le 23 novembre 2003 sur France 2. Le choix de ce corpus tient au caractère exemplaire du primat de l'ethos sur d'autres stratégies argumentatives que l'on peut y observer. Mais avant d'entamer l'analyse proprement dite, il convient d'abord de présenter l'approche méthodologique adoptée.

\section{Démarche méthodologique pour l'analyse de l'ethos en interaction}

2 La définition de l'ethos qui est retenue ici est celle proposée par Ruth Amossy. Il s'agit de l'« image de soi que le locuteur construit dans son discours pour exercer une influence sur son allocutaire» (Amossy, 2002 : 238). Cette définition doit évidemment se modeler en fonction du genre de discours considéré. Dans le cas du discours en interaction, la question de l'ethos doit être traitée dans sa dimension interactive car l'image de soi n'est pas le fait du seul locuteur, mais elle est co-construite par tous les participants à l'interaction. Amossy note à ce sujet que

la fonction de l'image de soi et de l'autre qui se construit dans le discours se manifeste pleinement dans cette perspective interactionnelle. Dire que les partenaires inter-agissent, c'est supposer que l'image de soi construite dans et par le discours participe de l'influence mutuelle qu'ils exercent l'un sur l'autre. (Amossy, 1999: 12)

3 Le locuteur est donc amené, tout au long de l'interaction à (ré)ajuster son ethos en fonction des répliques et des différents actes de langage que les autres interactants émettent à son égard. De ce fait, afin de rendre compte des images discursives mises en scène dans un discours en interaction, il faut les saisir dans cette dynamique d'actionréaction qui se noue entre les interactants.

Par ailleurs, nous supposons que les images construites peuvent être de deux sortes: valorisantes ou dévalorisantes. Les marqueurs de ces images varient selon que le locuteur se voit affublé d'un ethos défavorable ou qu'il s'auto-promeut et tente de donner une image favorable de lui-même.

Dans le premier cas, l'analyse se focalise notamment sur :

La qualification péjorative portant sur sa compétence, sa personnalité ou son caractère moral ;

Le recours à un vocabulaire péjoratif et dépréciatif lorsqu'il s'agit de s'exprimer sur des sujets inhérents à la personnalité ou à la vision du monde de l'interlocuteur ;

La formulation d'actes assertifs de critique et de reproche car ils véhiculent souvent, en creux, une image dévalorisante dont la nature est déterminée par la nature de l'objet sur lequel portent ces deux actes de langage ;

La formulation d'actes de langage menaçants à la face positive ${ }^{1}$ ou négative ${ }^{2}$ de l'interlocuteur tels l'ordre, l'injonction, la requête, qui permettent au locuteur de vexer son vis-à-vis, de le réduire à une position de dominé et de renvoyer, de lui, une image de docilité.

La mobilisation d'arguments ad hominem («argument de culpabilité par association », " argument d'avoir deux fers au feu», etc.) dont l'utilité est moins de contester la validité des arguments avancés par l'interlocuteur que de mettre en cause la cohérence de ce dernier, et, par conséquent, de porter atteinte à sa crédibilité ; 
6 Dans le second cas, l'analyse porte sur la revendication d'une image positive et les actes de langage valorisants formulés par le locuteur. Il faut noter, toutefois, que cette image n'est pas toujours énoncée, le locuteur peut «[la] présente[r] sous forme indirecte, dispersée, souvent lacunaire ou implicite» (Amossy, 1999 : 136). L'ethos est, ainsi, montré. Il renvoie aux :

traits de caractère que l'orateur doit montrer à l'auditoire (peu importe sa sincérité) pour faire bonne impression : ce sont ses airs. [...] je dois signifier ce que je veux être pour l'autre. [...] l'orateur énonce une information et en même temps il dit je suis ceci, je ne suis pas cela. (Barthes, $1970: 212$ )

7 L'image de l'orateur n'étant pas réductible à sa communication verbale et pouvant être également émise par tout ce qui est «ton de voix, débit de la parole, choix des mots et arguments, gestes, mimiques, regard, posture, parure, etc.» (Declerq, 1993: 48), l'analyse doit donc tenir compte de cette multicanalité à travers laquelle l'ethos est projeté. Les différentes images mises en scène par les procédés verbaux mobilisés se verront ainsi davantage mises en valeur ou nuancées suivant le ton, l'intonation et les différents comportements mimo-posturo-gestuels qui accompagnent leur énonciation.

\section{La description de l'ethos discursif de l'interviewé}

8 L'analyse du corpus a permis de montrer que la construction de l'ethos dans une interview politique télévisée s'effectue selon deux modalités distinctes : l'interaction et la locution. D'une manière générale, l'ethos se construit à travers la modalité de l'interaction lorsque les images résultent des co-actions que les interactants exercent les uns sur les autres lors de l'échange verbal. La modalité de la locution, quant à elle, concerne les différents procédés discursifs par le biais desquels l'interviewé s'autopromeut et construit une image favorable de lui-même.

\section{Sur le plan de l'interaction}

9 A travers les actes de langage qu'il lui adresse, l'intervieweur est souvent amené à donner, d'une manière explicite ou implicite, une image positive ou négative de l'interviewé, et, inversement, celui-ci construit une certaine image de soi à travers la manière dont il réagit aux images que l'intervieweur lui attribue. Seront traitées, dans ce point, d'abord, les images attribuées par l'intervieweur à NS, puis les stratégies discursives dont ce dernier se sert pour réagir aux images dévalorisantes qui lui sont attribuées.

10 S'il est admis que le débat politique télévisé, en raison de sa dimension conflictuelle intrinsèque, constitue un terrain favorable à la dévalorisation de l'image de l'adversaire (Sandré, 2014), l'interview politique télévisée, en dépit de sa dimension coopérative, n'en favorise pas moins la construction d'une image dévalorisante de l'homme politique interviewé. En effet, l'analyse des interventions initiatives de J-B. P a permis de dévoiler un intervieweur plutôt critique et agressif. La majorité de ses tours de parole véhicule, en creux, un acte de langage menaçant (principalement la critique) permettant d'induire une image négative de NS. L'analyse de ces actes a mis au jour 7 images dévalorisantes.

11 Cependant, pour atténuer la virulence de la conflictualité de l'échange, ces images négatives sont toutes sous-entendues par la critique de la politique menée par NS dans 
le domaine d'immigration. De plus, J-B. P recourt toujours à des adoucisseurs dont le plus important est "la formulation indirecte de l'acte de langage" (KerbratOrecchioni, 1996 : 55) qui consiste à faire passer un acte de langage menaçant pour un autre moins, ou nullement menaçant. Il s'agit, dans la presque totalité des cas recensés, de l'acte assertif de critique modalisé en interrogation (6 occurrences), ou, en termes simples, de la question rhétorique qui, en plus de lui permettre d'amortir l'agressivité de l'acte de critique, donne à voir une image dévalorisante de l'interviewé.

Au-delà de la modalité de leur attribution, les 7 images dévalorisantes recensées peuvent être répertoriées dans deux catégories distinctes:

La première catégorie regroupe les images négatives discréditant les valeurs morales de l'interviewé (à 3 reprises). Il en est ainsi de l'image d'hypocrisie et d'inhumanité sous-entendues dans la deuxième intervention initiative de J-B. P :

4J-BP.h: alors sur l'immigration/ on va parler d'immigration/ (.) lorsque vous résumez votre politique/ vous dites en général/ (.) cette politique elle a deux piliers/ (.) FERMEté/ (.) et puis de l'autre côté/ humanité .h (0.6s) alors (.) depuis le 28 octobre dernier/ la france a une nouvelle loi/ sur l'immigration/ (.) certes cette loi a aboli la double peine/ (0.4s) .h mais dans le même temps/ (0.4) lorsqu'on rend plus difficile/ (.) le (.) regroupement familial/ (.) >c'est-à-dire la possibilité pour un étranger/ de faire venir/ 4 (0.4s) sa femme/ ou ses enfants/ (0.4s) lorsque/ (.) on multiplie/ (0.4) .h les critères/ et les contrôles/ (0.45s) .h sur les mariages/ euh euh d'étrangers en france/ (0.5s) lorsqu'on allonge/ à trente jours/ le délai de rétention/\#\#

5 NStrrente deux

$6 \mathrm{~J}-\mathrm{BP} \rightarrow$ trente deux mainten- eh de $:$ : rétention donc des : des étrangers/ (.) aux frontières / (0.65S) -où est l'humanité là-'dans/

14 L'image d'hypocrite que l'intervieweur donne de NS, dans ce passage, est induite par l'emploi d'un type particulier d' « argument ad hominem circonstanciel »3 (Gauthier, 1995 : 174), à savoir « l'argument du tartuffe » (Ibid. : 177) qui vise «à mettre en évidence la contradiction entre un "dire" et un "faire", [...] à faire reproche à un locuteur d'adopter une forme de comportement incompatible avec le discours qu'il tient.» (Ibid.). La contradiction que J-B. P met, ici, en évidence est celle qui découle de ce que NS affirme de sa politique d'immigration : <le dire> " vous dites en général : "cette politique, elle a deux piliers : fermeté, et puis, de l'autre côté, humanité" »; et la façon dont cette politique est réellement mise en œuvre: <le faire> 1 . «on rend plus difficile le regroupement familial [...] », 2. « on multiplie les critères et les contrôles sur les mariages d'étrangers en France", 3. "on allonge à 32 jours le délai de rétention des étrangers aux frontières ». Ces trois énoncés $(1,2,3)$, dont le sujet, indéfini « on », renvoie sans conteste à NS, constituent des prémisses conduisant à la conclusion nécessaire que l'intervieweur formule sous forme de question rhétorique: «où est l'humanité làdans ? » qui tient lieu d'acte assertif négatif qui peut être reformulé ainsi : «il n'y a pas d'humanité là-dans ». En relevant une incompatibilité entre les dires de NS et sa pratique effective, qui est paradoxale avec ces dires, l'intervieweur l'affuble d'une image d'hypocrite.

Dans le même ordre d'idée, en mettant en cause la crédibilité de l'interviewé en dévoilant son hypocrisie, J-B. P met en valeur, par la même occasion, l'inhumanité de ce dernier car le dire auquel NS ne se serait pas conformé est précisément la mise en pratique des valeurs humanistes dont il prétendait que sa politique d'immigration soit imprégnée. Or, si la réalisation de cette politique ne tient aucun compte des valeurs humaines, voire les réprime (répression du droit des étrangers de vivre en famille, de 
se marier en France et d'être traité, pour les immigrés clandestins retenus aux frontières, dans des conditions décentes.), il est clair, selon J-B. P, que cette politique est dépourvue d'humanité. Etant l'auteur et le meneur de cette politique, NS reçoit, par ricochet, la qualification négative portée sur ladite politique. La qualification «cette politique est dépourvue d'humanité», qui ressort de la question rhétorique de l'intervieweur, semble ainsi conduire, suivant une logique de transfert, vers la conclusion : " vous êtes dépourvu d'humanité ».

Par ailleurs, si la force illocutoire des deux images dévalorisantes d'hypocrisie et d'inhumanité résulte de leur démonstration logique et des actes de justification qui les sous-tendent, elle semble, toutefois, contrebalancée par deux adoucisseurs : mis à part la formulation indirecte de l'acte de langage assertif de critique (modalisé, en l'occurrence, en interrogation), l'intervieweur fait également recours à :

un «désactualisateur personnel» (Kerbrat-Orecchioni, 1996: 56) en remplaçant la référence directe à l'intervieweur (vous) par l'emploi de l'indéfini : «lorsqu'on rend plus difficile [...], lorsqu'on multiplie [...], lorsqu'on allonge [...] ».

l'acte de concession introduit par «certes » dans «certes, cette loi a aboli la double peine », qui, en reconnaissant une mesure qui va dans le sens de la défense des valeurs humaines, minimise la dimension inhumaine de la politique pratiquée par NS en matière d'immigration.

La deuxième catégorie des images dévalorisantes formulées par l'intervieweur est associée aux actes de critique qui mettent en cause la compétence de l'interviewé en ce qui concerne sa gestion des affaires inhérentes au domaine d'immigration (à quatre reprises). En voici deux exemples obéissant, à peu de différences près, à une même stratégie de dévalorisation que celle mise en œuvre dans l'exemple précédent :

Extrait (1)

18J-BP $=$ d'accord $\backslash$ mais (.) en mettant comme ça autant de restrictions/ (.) de contrôles/ (.) est-c'qu'i n'y a pas PARAdoxalement/ (0.35s) le risque/ de $\Delta$ DEvelopper $\boldsymbol{\Delta}$ / l'immigration clandestine $/=$

Extrait (2)

22a J-BP mais je voudrais revenir sur sangatte $\backslash$ donc sangatte/ (.) vous l'avez fermé/ i y a un an $\backslash$ (.) eh quasiment $\backslash(0.4 s)$ or quand même/ (.) depuis/ (.) il y a des gens qui errent/ (0.3s) entre boulogne/ ouistreham/ (.) [dieppe]/\& 23NS [oui ]

22bJ-BP \&par exemple/ (0.27s) et (0.4s) là (.) finalement/ - est-c'que vous dites aujourd'hui/ c'était une BONNE mesure/ que de fermer sangatte 4 / =

L'intervieweur recourt ici à une stratégie interactionnelle reposant sur la mise en contraste de deux faits divergents : a) une action entreprise (extrait (1) : « en mettant comme ça autant de restrictions, de contrôles »; extrait (2) : «Sangatte, vous l'avez fermé il y a un an, quasiment ») ; b) et les conséquences de la mise en application de cette action (extrait (1) : « il y a paradoxalement le risque de développer l'immigration clandestine »; extrait (2) : «il y a des gens qui errent entre Boulogne, Ouistreham et Dieppe par exemple »). En mettant en parallèle, d'un côté, l'action et, de l'autre, ses répercussions dont il souligne, à travers ce qu'induit la question rhétorique et l'adverbe « paradoxalement » dans le premier extrait, et le connecteur d'opposition « or » dans le deuxième, qu'elles sont négatives, l'intervieweur laisse ainsi entendre que les mesures entreprises par NS ont abouti à des résultats contraires à ceux escomptés, ce qui témoigne de l'inefficacité de ces mesures, et donc de l'incompétence de leur initiateur. 
19 En ce qui concerne le degré de virulence de cette image dévalorisante, il faut noter que les mêmes procédés d'adoucissement de l'agressivité de l'attaque, que ceux mis en évidence ci-haut, ont été utilisés ici :

dans le premier extrait, il s'agit de la modalisation en interro-négation de l'acte assertif de critique qui peut être reformulé ainsi: «il y a paradoxalement le risque de développer l'immigration clandestine »; et l'énonciation délocutive qui, en faisant l'économie de l'indice personnel de l'allocutaire-cible de l'attaque, permet à l'intervieweur de ne pas mettre en cause directement NS.

dans le deuxième extrait, J-B. P recourt au même procédé d'atténuation de l'acte de critique à travers sa modalisation en interrogation («est-ce que c'était une bonne mesure que de fermer Sangatte ?») qui, compte tenu de l'argument avancé en amont de cette interrogation contre la mesure prise par NS ( or quand même, depuis...par exemple »), vaut pour : «ce n'était pas une bonne mesure que de fermer Sangatte ».

La stratégie réactive adoptée par NS face aux images dévalorisantes dont il se voit affublé est, tel qu'il ressort de l'analyse de ses interventions réactives, celle de l'autodéfense. Cette stratégie se traduit, sur le plan discursif, par le recours aux actes d'explication et de justification visant à contrer la critique adressée par l'intervieweur en recadrant son objet de telle sorte que l'image dévalorisante qui en découle devienne caduque, ou en avançant des justifications et des contre-arguments allant à l'encontre des arguments qui la sous-tendent. Il en est ainsi de la stratégie qu'il a déployée pour parer à l'image d'inhumanité et celle d'hypocrisie que l'intervieweur donne de lui :

15 NS [...] et <((l'air étonné)) je ne comprends pas/ (0.7s) qu'est-c' qu'il y a de contraire aux droits de l'homme/ (0.4s) que d'ramener/ (0.27s) un roumain en roumanie/ (0.25s) un bulgare en bulgarie/ (.) un sénégalais au sénégal/> $(0.36 \mathrm{~s}) \mathrm{s}^{\prime} \mathbf{i}$ n'ont pas d'papiers/ (0.53s) i n'ont PAS le droit d'se maintenir $\backslash(0.25 \mathrm{~s})$ c'est vrai/ (0.32s) que j'ai durci/ (0.4s) la loi sur l'immigration\ (0.4s) mais j'l'ai fait pourquoi/ (0.77s) parc'que (.) nous n'pouvons pas être le SEUL/ pays au mon : :de/ (0.5s) qui n'ait pas le droit d'décider/ de qui rentre sur notre territoire/\#\#

16J-BP mais/\#\#

$17 \mathrm{NS} \rightarrow$ et de qui n'a pas à s'y maintenir $/=$

NS commence, d'abord, par réfuter toute relation d'implication entre le fait de renvoyer un immigré clandestin dans son pays d'origine et celui de porter atteinte à ses droits d'être humain. La remise en question de l'association entre ces deux faits se laisse clairement saisir par l'étonnement accompagnant l'énonciation de cet énoncé (" et je ne comprends pas...»). Ce faisant, NS rejette déjà l'image d'inhumanité et, cela va de soi, d'hypocrisie que l'intervieweur donne de lui.

De plus, il faut noter que la stratégie de réaction de NS est d'autant plus subtile qu'elle recourt à un recadrage de l'objet sur lequel porte la critique de l'intervieweur. Au moment où ce dernier qualifie d'inhumain la politique d'immigration de NS, celui-ci recadre l'objet de la critique en y substituant un élément nullement condamnable, à savoir celui d'appliquer rigoureusement la loi : « s'ils n'ont pas de papiers, ils n'ont pas le droit de s'y maintenir ».

Une fois la critique de la politique répressive qui lui est adressée est réorientée vers celle de l'application au pied de la lettre de la loi, NS renforce davantage sa stratégie réactive par l'emploi d'un acte de justification lui permettant d'expliquer la raison d'une telle rigueur : «mais je l'ai fait pourquoi? parce que nous ne pouvons pas être le seul pays au monde qui n'ait pas le droit de décider de qui rentre sur notre territoire et 
de qui n'a pas à s'y maintenir ». L'interviewé assoit la justification du durcissement de sa politique sur le topos selon lequel « il faut suivre la règle commune».

Notons, en définitive, que, face aux cinq autres images négatives dont il se voit affublé au cours de l'interview, la stratégie de réaction adoptée par NS oscille entre l'un et l'autre des deux procédés d'autodéfense que nous venons de décrire, c'est-à-dire tantôt en recadrant le contenu dévalorisant véhiculé par l'acte de critique dont il constitue la cible, tantôt en mobilisant des actes de justification allant à l'encontre des arguments avancés par l'intervieweur.

En somme, en réagissant par l'auto-défense aux nombreuses images dévalorisantes qui lui sont attribuées, NS fait preuve de retenue et du sens de la mesure, un ethos dont il doit être imprégné en sa qualité de $2^{\text {ème }}$ homme de l'état. Ceci dit, s'il se défend de se montrer agressif en n'attribuant aucune image dévalorisante à son interlocuteur, il ne se montre pas moins résistant en déployant une stratégie de défense aussi efficace qu'inoffensive dans la mesure où elle lui permet de contrecarrer l'image dévalorisante imposée tout en évitant de porter atteinte aux faces positive et négative de l'intervieweur.

\section{Sur le plan de la locution}

Afin de gagner la confiance des téléspectateurs, le locuteur peut opter pour la stratégie de l'autopromotion. Il s'agit d'une auto-valorisation qui se traduit par la mobilisation d'acte de promotion, de promotion de sa propre personne, de ses compétences, de ses valeurs morales, etc.

L'analyse a permis de constater l'utilisation de deux formes d'autopromotion : celle où le locuteur revendique implicitement une image valorisante; et celle où l'image positive est projetée à travers le discours. 11 images valorisantes auto-attribuées sont ainsi recensées. Plus précisément, NS préfère confier au discours la tâche de son autopromotion: 9 images valorisantes s'y inscrivent, alors que seules deux images positives sont revendiquées plus ou moins explicitement.

Etant donné que les images dévalorisantes que l'intervieweur lui impose visent à le discréditer sur deux plans distincts, à savoir celui de ses valeurs morales et celui de sa compétence, en plus d'y réagir en s'auto-défendant, NS tente également de contrer ces images en y opposant des images antinomiques et en s'auto-valorisant sur ces deux plans précis.

29 Il sera question, dans ce qui suit, de la description, dans un premier temps, des images revendiquées, et dans un second temps, des images montrées par NS.

Les deux seules images que NS revendique clairement, au cours de l'interview, sont celle d'ouverture vers l'autre et celle d'efficacité. Le choix de ces deux images est d'autant plus pertinent que l'incompétence et la xénophobie de NS figurent parmi les points ciblés par les actes de critique qui lui sont adressés.

31 Aussi, afin de contrer la critique mettant en cause sa compétence notamment dans la gestion du problème de l'immigration clandestine, NS rappelle et fait l'éloge de ses accomplissements en la matière. Il le fait :

tantôt en comparant son bilan, décrit comme positif, à celui de ses prédécesseurs, qui est présenté implicitement comme moins favorable : 
19a NS [...] lorsque j'suis devenu ministre de l'intérieur/ (1s) dans les zones de rétention d'roissy/ (0.6s) c'était CINQ CENTS clandestins par jour $\backslash(0.8 \mathrm{~s})$ nous avons divisé par CINQ/ ce nombre $\backslash$ (.) [...]

tantôt en le mettant en parallèle avec les objectifs escomptés de la mise en œuvre de sa politique de lutte contre l'immigration clandestine :

Extrait (1)

19a NS [...] depuis qu'j'ai fermé sangatte/ (0.7s) qui avait été CONFORtablement installé là-bas/ (0.5s) le nombre de clandestins dans l'calaisie/ (0.23s) -a été divisé par dix $4 /$

Extrait (2)

32a NS [...] d'abord je crois à la coopération internationale $\backslash(0.6 \mathrm{~s})$ avec la roumanie ça marche/ (0.8s) ๔on a pu raccompagner la moitié des roumains/ en situation irrégulière / $(0.7 \mathrm{~s})$ avec la bulgarie ça marche/ $(0.4 \mathrm{~s})$ notalement/ $(0.3 \mathrm{~s})$ sur le démantèlement de VINGT CINQ réseaux de proxénètes/ [...]

32 A l'image de xénophobie que l'intervieweur laisse entendre de lui à travers sa critique du traitement inhumain qu'il offre aux étrangers clandestins, NS substitue celle d'ouverture vers l'autre. Ainsi affirme-t-il :

7 NS [...] je crois qu'la france a besoin/ (0.9s) de recevoir/ (0.65s) des étrangers/ (0.85s) je crois qu'une SOciété qui se referme sur elle/ $(0.4 \mathrm{~s})$ une société consanguine/ (0.35s) est une société et une civilisation/ (.) qui meurt $\backslash[.$.

la prédominance du discours verbal sur le discours nominal, ce qui «donne l'impression de dynamisme et du mouvement " (Calvet et Véronis 2008 : 37, cité dans Pigliapochi, 2010 : 125). La majorité des verbes d'action utilisés étant accompagnés de la forme nominale d'auto-désignation « je », NS se montre donc engagé dans l'action et empreint par ce dynamisme.

la mise en avant de sa détermination à résoudre les problèmes liés à l'immigration clandestine, et des efforts fournis pour y arriver :

Extrait (1)

19a NS [...] je me bats $($.$) pied à pied/ (.) [...]$

Extrait (2)

$32 \mathrm{~b}$ NS [...] moi j'essaie de faire une chose/ (0.25s) c'est d'grimper la montagne\

(.)\#\#

$34 \mathrm{~J}$-BP ce je [ra- ]\#\#

35 a NS $\rightarrow[$ [ pour $][$ essayer $] \&$

36 OM [alors ]\#

35b NS \&d'résoudre les problèmes/\#\#

L'image d'engagement, qui n'est que celle d'action projetée dans l'avenir. Le marqueur le plus important de cette image est l'utilisation des formes nominales d'autodésignation telles que " je » et " nous ", suivies de verbes d'action conjugués à un temps $\mathrm{du}$ futur, introduisant les objectifs que NS se propose d'atteindre, comme dans les deux exemples ci-dessous :

Extrait (1)

7 NS [...] j'ai fixé un objectif/ (1S) nous raccompagnerons chez eux/ (0.65s) DEUX

fois plus de clandestins/ (0.5s) que c'qui a été fait/ (.) jusqu'à présent $\backslash(0.76 \mathrm{~s}) \# \#$

Extrait (2) 
27b NS [...] mais croyez bien/ (0.25s) c'est ma PRIOrité \(0.6s) et j'obtiendrai des REsultats en la matière/

L'image de lucidité et de maîtrise qui se donne à voir à travers l'utilisation récurrente (à 4 reprises) de " la justification par le poids des circonstances $»^{4}$ (Charaudeau, 2005 : 36) tel dans les deux exemples ci-dessous où NS se montre conscient de l'aggravation du problème d'immigration clandestine, et souligne l'urgence d'y apporter des solutions, ce qui lui permet de justifier les mesures qu'il a entreprises pour y parvenir (politique répressive en matière de lutte contre l'immigration clandestine) :

Extrait (1)

19a NS [...] la situation ne peut plus durer comme cela/ (0.29s) pourquoi la France/ $(0.27 \mathrm{~s})$ serait-elle à frontières ouvertes/ $(0.35 \mathrm{~s})$ c'est quand même pas (0.25s) notre rôle/ (0.32s) Tous les autres pays dans le monde/ font ça/ [...]

Extrait (2)

27b NS [...] mais croyez bien/ (0.25s) c'est ma PRIOrité \(0.6s) et j'obtiendrai des REsultats en la matière/ $(0.35 \mathrm{~s})$ comme $\mathrm{j}$ 'ai essayé d'les obtenir/ en matière de sécurité \la situation (.) ne pouvait plus/ (0.25s) durer (0.3s) de la sorte $>=$

Au-delà des procédés permettant la projection de ces trois images d'action, d'engagement, et de maîtrise de la situation, il faut noter que leur choix est loin d'être fortuit. Ces trois images permettent, d'une part, de contrecarrer l'image dévalorisante d'incompétence dont l'intervieweur tente de l'affubler, et, d'autre part, de consolider son ethos préalable en faisant correspondre sa présentation de soi à l'image d'homme d'action, de « l'homme pressé » qu'on lui attribue généralement dans l'espace public.

Une autre image qui vise à rappeler l'ethos préalable de NS, en particulier celui de "père fouettard » associé à la fonction du ministre de l'Intérieur qu'il exerce, est celle d'autorité et de fermeté qui, étant en l'occurrence appropriée, d'un côté, à son statut socioprofessionnel et, de l'autre, à la dévalorisation entreprise par l'intervieweur à son égard, se présente comme valorisante. Quant à sa mise en scène, elle se manifeste à travers le recours :

à l'évocation de sa rigueur dans l'application de la loi, comme l'illustrent les deux exemples ci-dessous :

Extrait (1)

7 NS [...] les étrangers avec des papiers/ sont les bienvenus/ (0.55s) les étrangers SANS papiers/ ou avec des FAUX papiers/ (0.35s) seront raccompagnés chez eux\ [...]

Extrait (2)

15 NS [...] lorsqu'i ya une DECIsion/ (0.6s) de reconduite/ (.) à la frontière d'un étranger en situation (.) IRREgulière/ (0.4s) elle sera/ (.) exécutée $\backslash[. .$.

à la formulation, à l'endroit de l'intervieweur, de quelques actes de langage vexatoires, tel le micro-acte assertif-rectificatif dans :

$4 \mathrm{~J}-\mathrm{BP}$ [...]lorsqu'on allonge/ à trente jours/ le délai de rétention/\#\#

5 NS trente deux

$6 \mathrm{~J}-\mathrm{BP} \rightarrow$ trente deux mainten- eh de : :

à l'attaque récurrente (à quatre reprises) des tiers-adversaires comme dans :

Extrait (1)

15 NS [...] parc'que qui ne voit/ (0.5s) que le laxisme/ invraisemblable/ de toutes ces dernières années/ (0.43s) a conduit à la montée de l'exaspération/ $(0.6 \mathrm{~s}) \mathrm{du}$ racisme/ (0.5s) et de l'amalgame $\backslash[. .$.

Extrait (2)

24a NS [...] j'ai été à sangatte\(0.5s) voyez vous qu'j'ai participé à moins d'colloques/ sur les droits de l'homme/ (0.45s) que madame aubry/ (0.3s) ou 
madame guigou/ (0.33s) mais moi j'ai été quatre fois à sangatte/ (0.45s) elles jamais $\backslash(\mathbf{0 . 4 5 s})$ alors qu'elles sont des élues/ (0.33s) de la région $\backslash(0.25 \mathrm{~s})$ je n'leur fais pas le procès pour cela/ $(0.35 \mathrm{~s})$ mais de là à nous donner des leçons d'humanité/ quand même/

Extrait (3)

$32 \mathrm{~b}$ NS alors on me dit c'est trop peu/ (0.35s) mais ceux qui me disent c'est trop peu/ (0.23s) qu'est c'qu'i z ont fait avant/ (0.25s) rien\ « tous les problèmes » en témoigne :

Extrait (1)

19a NS [...] je n'dis pas monsieur prédali/ (0.3s) que TOUT a été réglé/ (0.25) c'est TELLement difficile/ [...]

Extrait (2)

27a NS [...]je n'p- dis pas que je peux régler à moi tout seul/ (0.35s) en dix-neuf mois/ (0.3s) tous les problèmes d'immigration du mon :de/

Extrait (3)

39 NS $\rightarrow$ naturellement qu'on n'résout pas tout/\#\# 


\section{sont :}

l'utilisation des procédés explicatifs comme :

L'illustration par l'exemple :

19a NS au contraire/ ( $0.65 \mathrm{~s}) \mathrm{j}$ 'en prends un exemple très simple/ (0.45s) lorsque j'suis devenu ministre de l'intérieur/ (1s) dans les zones de rétention d'roissy/ (0.6s)

c'était CINQ CENTS clandestins par jour $\backslash[. .$.

46

L'énumération gestuelle à travers le comptage par l'index d'une main touchant le pouce, puis l'index, et enfin le majeur de l'autre main, comme dans la Figure 1 cidessous :

Figure 1 : Enumération gestuelle

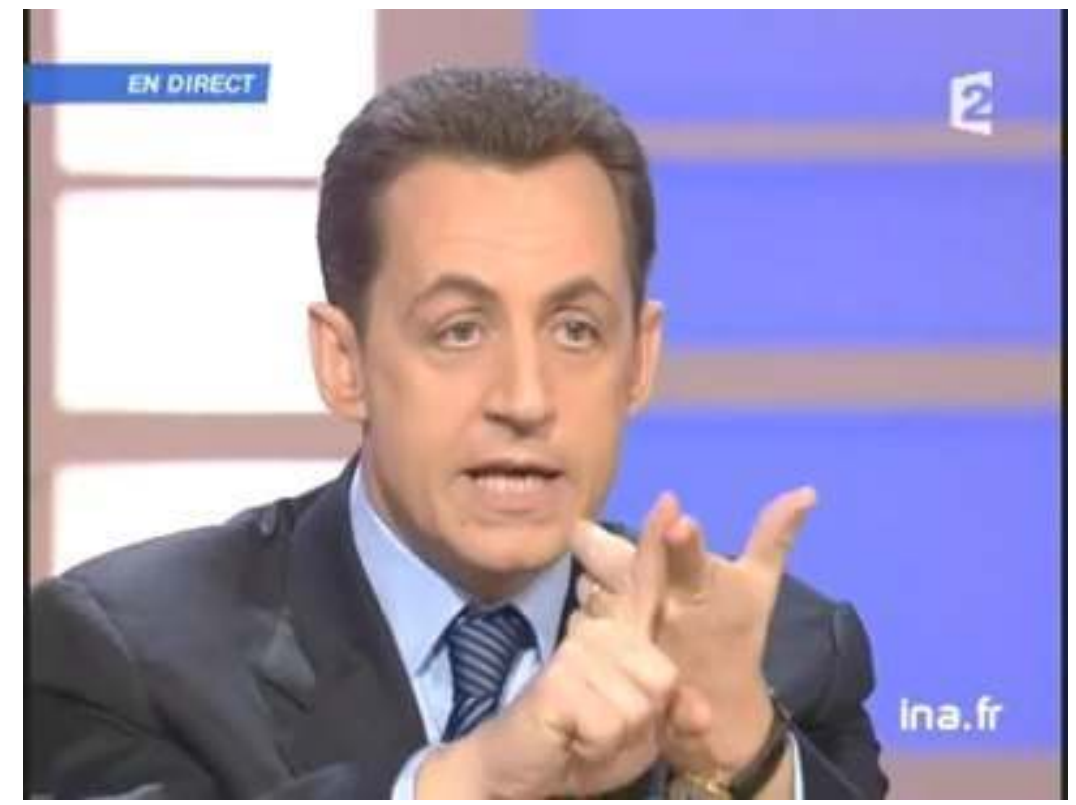

le souci de précision qui se traduit par l'emploi abondant de chiffres accompagnés d'un geste coverbal approprié à cet effet, en l'occurrence la pince digitale. Ce geste accompagne, dans le discours de NS, presque régulièrement l'évocation de chiffres :

19a NS au contraire/ (0.65s) j'en prends un exemple très simple/ (0.45s) lorsque

j'suis devenu ministre de l'intérieur/ (1s) dans les zones de rétention d'roissy/ (0.6s)

c'était

*CINQ CENTS* clandestins par jour $\backslash$ (0.8s) nous avons divisé par CINQ/ ce nombre\

*pince digitale* 
Figure 2 : pince digitale sur « cinq cents »

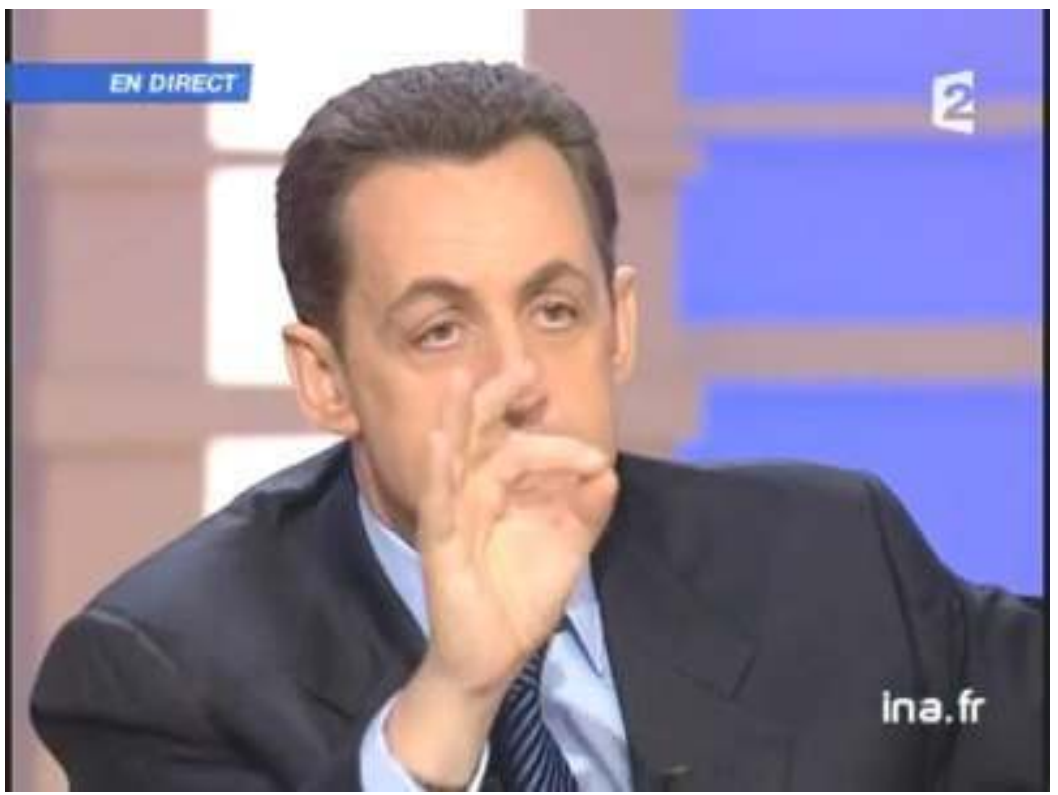

Le recours aux questions rhétoriques comme dans les deux exemples ci-dessous :

Extrait (1)

15 NS [...] $\boldsymbol{\Delta}$ je souhaite/ (.) POURSUIvre $\mathbf{\Delta /}$ (.) dans les années suivantes $\backslash($. pourquoi/ (.) monsieur prédali/ (0.66s) parc'que qui ne voit/ (0.5s) que le laxisme/ invraisemblable/ de toutes ces dernières années/ (0.43s) a conduit à la montée de l'exaspération/ (0.6s) du racisme/ (0.5s) et de l'amalgame \[...]c'est vrai/ (0.32s) que j'ai durci/ (0.4s) la loi sur l'immigration\(0.4s) mais j'l'ai fait pourquoi/ (0.77s) parc'que (.) nous n'pouvons pas être le SEUL/ pays au mon : :de/ (0.5s) qui n'ait pas le droit d'décider/ de qui rentre sur notre territoire/\#\#

Extrait (2)

19a NS [...] nous avons divisé par CINQ/ ce nombre $\backslash$ (.) pourquoi/ (0.8s) parce que les réseaux savent bien/ qu'on n'passe plus/ [...]

l'emploi d'un style verbal proche de l'oral :

Un vocabulaire familier :

7 NS .h monsieur prédali/ c'est très simple/ (.) les français/ doivent le savoir/ la France n'avait plus d'politique d'immigration\(1.1s) c'était devenu n'importe quoi/

Elision du « e » final de « de », « que », « le », etc. :

$7 \mathrm{NS}[. .$.$] la France n'avait plus d'politique d'immigration \backslash[. .$.$] mais je veux vous$ l'dire/ [...] nous raccompagnerons chez eux/ (0.65s) DEUX fois plus de clandestins/ (0.5s) que c'qui a été fait/ (.) jusqu'à présent $\backslash(0.76 \mathrm{~s}) \# \#$

Contraction du pronom démonstratif « cela » en « ça », utilisé surtout à l'oral :

12 NS ça nous amènera/ (0.3s) à vingt milles/ (0.3s) expulsions/

Il ressort de l'analyse de la construction de l'ethos sur le plan de la locution que NS multiplie autant les images valorisantes qu'il s'auto-attribue que les procédés discursifs qui entrent dans leur construction. La modalité de la locution est, décidément, celle à laquelle l'interviewé recourt le plus pour construire son ethos en situation d'interview. En témoigne notamment le nombre important d'images valorisantes qu'il s'autoattribue au cours de l'interaction. Cette proportion peut s'expliquer par la nécessité de 
substituer aux images dévalorisantes que l'intervieweur lui attribue d'autres, favorables, et à même de séduire les téléspectateurs.

En définitive, l'analyse de l'ethos, en situation d'interview politique télévisée, nous permet de conclure que sa mise en scène met à contribution tous les canaux de la communication orale. L'ensemble des comportements verbaux, paraverbaux et nonverbaux concourt concomitamment à la construction de l'ethos de l'interviewé. Par ailleurs, sa projection dans le discours est de nature multimodale : elle emprunte à la fois la modalité de l'interaction et celle de la locution. Nous avons pu montrer également que, contrairement à ce qu'on pourrait penser apriori, l'interview, en dépit de son caractère coopératif, n'exclue pas pour autant une présentation dévalorisante de l'homme politique interviewé. De ce point de vue, elle se rapproche des genres de discours polémiques tel que le débat. Néanmoins, contrairement à ce dernier, où la dimension conflictuelle est clairement affichée, le contrat de communication de l'interview, trop rigide, contraint l'intervieweur à enrober d'adoucisseurs et de précautions interactionnelles les attaques qu'il adresse à l'interviewé. L'ethos discursif de celui-ci se construit ainsi dans cette dynamique interactionnelle. Il émane de la confrontation entre les tentatives de dévalorisation entreprises par l'intervieweur et les actes d'autopromotion mis en œuvre par l'interviewé. Ses marqueurs deviennent alors aussi divers que variés. Sa mise en scène résulte de la mobilisation de plusieurs procédés discursifs dont notre étude s'est proposé de rendre compte.

\section{BIBLIOGRAPHIE}

AMOSSY, Ruth, «L'ethos au carrefour des disciplines : rhétorique, pragmatique, sociologie des champs ", dans Images de soi dans le discours. La construction de l'ethos, AMOSSY RUTH (dir.), Editions Delachaux et Niestlé, Lausanne, 1999, pp. 129-154.

AMOSSY, Ruth, entrée « Ethos ", dans Dictionnaire d'analyse du discours, CHARAUDEAU PATRICK et MAINGUENEAU DOMINIQUE (dirs.), Seuil, Paris, 2002, p. 238.

BARTHES, Roland, «L'Ancienne Rhétorique », Communications, volume 16, 1970, pp. 172-223. Disponible sur le site : http://www.persee.fr/doc/comm_0588-8018_1970_num_16_1_1236

CALVET, Louis-Jean et VÉRONIS, Jean., Les Mots de Nicolas Sarkozy, Seuil, Paris, 2008.

CHARAUDEAU, Patrick, «Quand l'argumentation n'est que visée persuasive. L'exemple du discours politique », inBURGER M. et MARTEL G (dirs), Argumentation et communication dans les médias, Éditions Nota Bene, Québec, 2005.Disponible sur le site : http://www.patrickcharaudeau.com/Quand-l-argumentation-n-est-que.html

DECLERCQ, Gilles, L'art d'argumenter. Structures rhétoriques et littéraires, Editions Universitaires, Paris, 1993.

GAUTHIER, Gilles, « L'argumentation périphérique dans la communication politique : le cas de l'argument "ad hominem" », Hermès 16, Argumentation et rhétorique, 1995, pp. 167-185.

Disponible sur le site : http://documents.irevues.inist.fr/handle/2042/15190 
KERBRAT-ORECCHIONI, Catherine, « Rhétorique et pragmatique : les figures revisitées », Langue française, $n^{\circ}$ 101, 1994, pp. 57-71. Disponible sur le site : <http://www.persee.fr/web/revues/ home/prescript/article/lfr_0023-8368_1994_num_101_1_5843>

KERBRAT-ORECCHIONI, Catherine, La conversation, Seuil, Paris, 1996.

NOWAKOWSKA, Aleksandra, « Du dialogal et du dialogique dans l'interview politique », Congrès Mondial de Linguistique Française - CMLF, 2012, pp. 613-628. Disponible sur le site : <http:// www.shs-conferences.org/articles/shsconf/pdf/2012/01/shsconf_cmlf12_000139.pdf>

PIGLIAPOCHI, Susanna, «La stratégie communicative de Nicolas Sarkozy », in Autres Modernités, $\mathrm{n}^{\circ}$ 3, Sezione di Studi Culturali, Università degli Studi di Milano, 2010, pp. 120-132. Disponible sur le site : http://riviste.unimi.it/index.php/AMonline/article/download/559/753

SANDRE, Marion, «Éthos et interaction : analyse du débat politique Hollande-Sarkozy », Langage et société, $\mathrm{n}^{\circ}$ 149, 2014/3, pp. 69-84. Disponible sur le site : https://www.cairn.info/revue-langageet-societe-2014-3-page-69.htm

\section{NOTES}

1. Elle «correspond en gros au narcissisme, et à l'ensemble des images valorisantes que les interlocuteurs construisent et tentent d'imposer d'eux-mêmes dans l'interaction » (KerbratOrecchioni, $1994: 65)$.

2. Elle regroupe le «territoire corporel, spatial ou temporel; biens et réserves matérielles ou cognitives.» (Ibid.). Une tentative d'irruption dans l'un ou dans l'autre de ces territoires personnels et intimes constitue une menace à la face négative de l'individu. Il en est ainsi, par exemple, d'une question indiscrète ou d'un acte d'excuse ou de confession.

3. Gauthier définit ce type d'argument comme une "tentative pour mettre en contradiction avec luimême un locuteur du fait d'une incompatibilité entre la position qu'il affiche et quelque trait de sa personnalité ou de son comportement » (Gauthier, 1995 : 175).

4. Il s'agit d'un type d'argument où, pour paraphraser Charaudeau, le locuteur répond à l'évocation d'une contrainte négative en proposant un moyen d'y remédier.

\section{RÉSUMÉS}

La présente étude traite de la construction de l'ethos dans un discours en interaction. Elle vise à rendre compte des modalités de mise en scène des images de soi et de l'autre en situation d'interview politique télévisée. Il s'agit précisément de mettre au jour les procédés discursifs à travers lesquels l'ethos de l'interviewé est projeté et de voir si, contrairement à ce que l'on pourrait penser a priori, l'interview peut constituer, à l'instar des genres de discours polémiques, un terrain favorable à la construction d'images dévalorisantes de l'homme politique interviewé. Pour ce faire, l'analyse s'appuie sur un appareil conceptuel et théorique emprunté à l'Analyse du Discours en Interaction et à la pragmatique linguistique.

The present study treats the ethos' construction in an interacting speech. It aims at describing the modes of staging images of oneself and the other in televised political interview situation. 
Precisely, it's about to raise the discursive markers through which the interviewed person's ethos is highlighted and to see if, contrarily to what one might think at first glance, the interview may constitute, like polemical speech, a convenient field to the construction of the interviewed politician's demeaning images. To do so, the analysis relies on a set of concepts and theories borrowed from Discourse Analysis in Interaction and linguistic pragmatics.

INDEX

إيثوس الخطابية, التفاعل, مقابلة سياسية متلفزة, فعل الكلام, صورفهرس الكلمات المفتاحية:

مثمنة ومهينة

Mots-clés : ethos discursif, interaction, interview politique télévisée, acte de langage, image valorisante et dévalorisante

Keywords : discursive ethos, interaction, televised political interview, speech act, advantageous and demeaning image

\section{AUTEUR}

\section{ZAKARIA TANSSAOUT}

Ecole Normale Supérieure LSH Bouzaréah 